\title{
Relapsing bronchiolitis obliterans organising pneumonia and chronic sarcoidosis in an atopic asthmatic patient
}

\author{
C. Carbonelli1 , A. Roggeri1 , A. Cavazza², M. Zompatori3, L. Zucchi1
}

\begin{abstract}
Relapsing bronchiolitis obliterans organising pneumonia and chronic sarcoidosis in an atopic asthmatic patient. C. Carbonelli, A. Roggeri, A. Cavazza, M. Zompatori, L. Zucchi.

Asthma is thought to be a Th2 disease while sarcoidosis is considered a Th1 granulomatous disorder. Organising pneumonia is a histologic pattern of lung injury. When it has no recognisable cause it is defined as cryptogenic organising pneumonia. We herein report the case of a patient with recurrent and steroid sensitive organising pneumonia
\end{abstract}

\begin{abstract}
associated with chronic sarcoidosis in an atopic, moderate persistent asthmatic patient. Each disease has been documented with transbronchial biopsies and recurrence of organising pneumonia was suggested by clinical features and by follow up HRCT which shows distinctive signs even in associated disease. Steroids are the mainstay of therapy for these disorders and especially for the consolidated processes typical of organising pneumonia but prognostic indices for relapse and progression are lacking.

Monaldi Arch Chest Dis 2008; 69: 1, 39-42.
\end{abstract}

Keywords: Bronchiolitis obliterans organising pneumonia, Sarcoidosis, Asthma, Recurrence, Computed tomography.

1 Department of Respiratory Diseases, Hospital Santa Maria Nuova, Reggio Emilia,

2 Department of Pathology, Hospital Santa Maria Nuova, Reggio Emilia,

3 Department of Clinical Sciences, Section of Radiology University of Parma, Italy.

Correspondence: Dr. Cristiano Carbonelli, UO Pneumologia Ospedale ASMN Re, V.le Risorgimento 80, 42100 Reggio Emilia, Italy; e-mail: carbonelli.cristiano@asmn.re.it

\section{Case report}

A 36-year-old man was admitted to the hospital in 2004 because of persistent cough without fever and with bilateral lung opacity particularly in the left lower lobe and enlargement of the hila on standard radiograph. His history showed sensitisation to seasonal aeroallergens (grass pollen) and moderate persistent asthma, treated with inhaled steroids and $\beta 2$ agonists on a regular basis. On examination he was noted to have a mild bronchospasm, and his vital signs were normal. High resolution computed tomography (HRCT) of the thorax revealed massive enlargement of the mediastinal subcarinal, paratracheal and left hilar lymph nodes, multiple bilateral nodules distributed subpleurally and along the broncovascular tree and an extensive consolidation with air bronchogram in the left lower lobe (see figure 1). The results of routine blood and urine tests revealed increased erythrocyte sedimentation rate $(78 \mathrm{~mm})$ and $\alpha 2$ globulin [16\% of the seroprotein (n.v. 8.2-13)], a mild reduction of the percentage of lymphocytes ( $10 \%$ of the blood white cells), increase of the alkaline phosphatase [520 U/l (n.v. 91-258)] and of the $\gamma$-glutamil transferase ( $\gamma$ GT) $[214$ U/l (n.v. 1050)]. There was a mild increase of total IgE [157 $\mathrm{Ul} /, \mathrm{ml}$ (n.v. 0-120)] and of angiotensin converting enzyme (ACE) [62 U/l (n.v. 18-55)]. ANA test, Waaler Rose, RF, ANCA were all negative. Spirometry showed a medium-marked obstructive pattern, $\mathrm{FEV}_{1} 63 \%$ of the predicted value, a reduction of the $\mathrm{FEV}_{1} / \mathrm{FVC}$ ratio at $49 \%$. DLCO/VA was slightly reduced but still in the normal range.

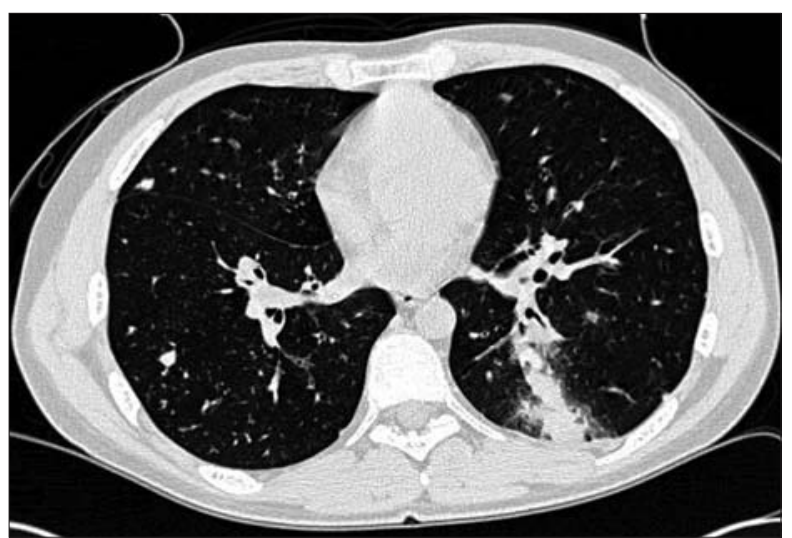

Fig. 1. - First CT examination: HRCT scan through the lung bases demonstrates peripheral non calcified nodules. A few of them are distributed in the subpleural regions. These nodules are compatible with the diagnosis of a granulomatous lung disorder, particularly active Sarcoidosis. A slight thickening of the bronchial walls and a large area of consolidation, located in the posterior segment of the left lower lobe was noted. Within the area of consolidation, the air bronchogram sign can be seen, along with a peripheral zone of intermediate (ground glass) density, surrounding the dense consolidation (halo sign). From the radiological viewpoint, a lesion like this one in a patient with suspected Sarcoidosis is suggestive of alveolar Sarcoidosis or superimposed disease process. In itself, however, this presentation is not specific and could be found in a large array of lung pathology, comprising acute bronchopneumonia or subacute-chronic processes, such as organising pneumonitis, bronchiolo-alveolar carcinoma and so on. 
There was no bronchodilator response. Bronchoscopy and bronchoalveolar lavage were performed. Thin secretions were noted and the sputum was not grossly purulent. The BAL-fluid analysis demonstrated 367.000 cells $\cdot \mathrm{mL}^{-1}$, with $35 \%$ macrophages, $25 \%$ neutrophils, $8 \%$ eosinophils, $32 \%$ lymphocytes with a slight reduction of $\mathrm{CD} 4 / \mathrm{CD} 8$ ratio (0.6). No bacteria, viruses or mycobateria grew on bronchoscopic cultures. Transbronchial biopsies of the lungs showed multiple lesions: bronchial histopatological changes typical of bronchial asthma, non necrotising well defined granulomas with a lymphangitic distribution suggesting sarcoidosis and plugs of alveolar organization typical of Organising Pneumonia (OP) (see figure 2 and 3). Stainings for fungi and mycobacteria were negative. Prednisone 50 $\mathrm{mg} /$ day was started and tapered to cessation in 8 months. There was a prompt resolution of the cough and clearing of HRCT areas of lung consolidation processes and an improvement of the interstitial micronodular and mediastinal involvement (see figure 4); spirometry showed a significant improvement of the obstructive defect.

In March 2005, a few weeks after steroid cessation, the patient complained of a recurring cough,

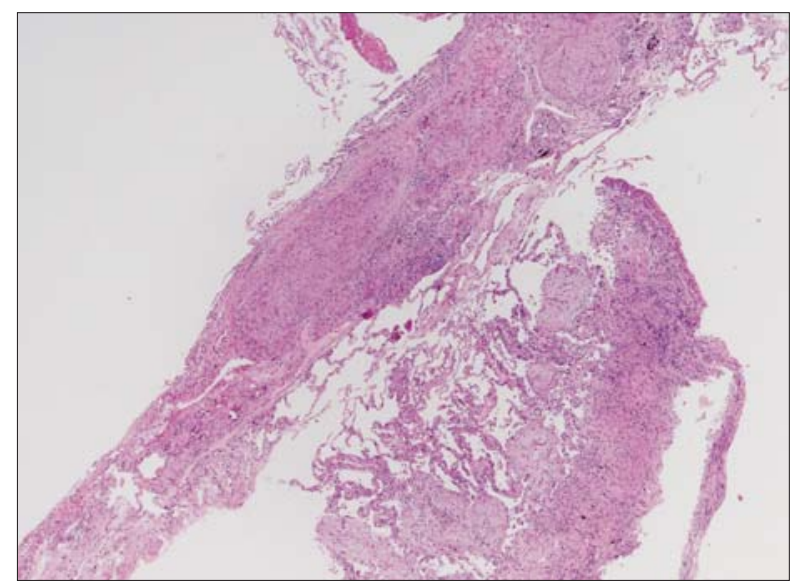

Fig. 2. - The transbronchial biopsy showed two lesions. The first (left) was represented by well-formed non necrotising granulomas associated with dense fibrosis, typical of sarcoidosis. The second lesion (right) consisted in a focus of organising pneumonia.

Hematoxylin-Eosin, 40x.

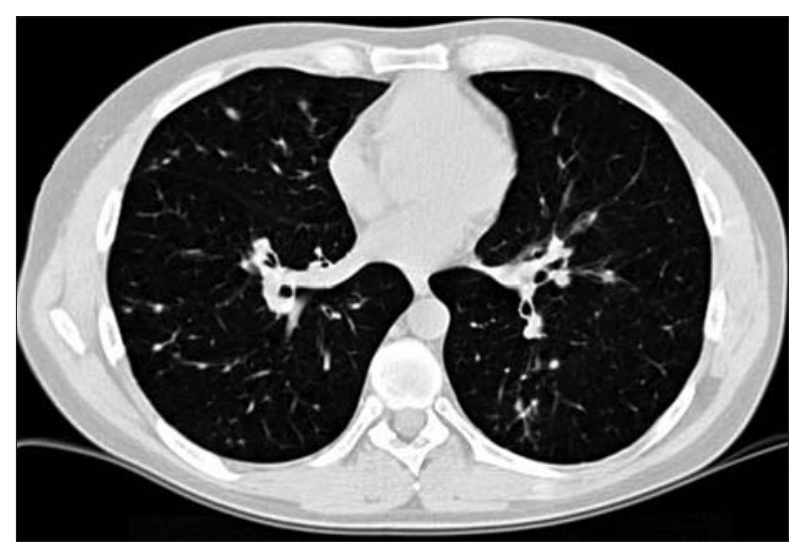

Fig. 4. - Second CT exam. HRCT scan at the same level shows a complete resolution of the consolidated area. A few, bilateral small nodules are still visible at the lung periphery. mild dyspnoea, low grade fever and bronchospasm. A new consolidated process in the right upper lobe was seen at chest X-rays. This clinical scenario recurred in November and was associated with another consolidated process in the apical right lower lobe segment. Functional obstructive impairments were demonstrated as well. In both manifestations the lack of radiological resolution after wide spectrum antibiotic courses, the compatibility of the HRCT appearance (see figure 5), and the dramatic reversibility especially of the consolidated processes with corticosteroids, led us to conclude that there were recurrences of previous condition. The results of routine blood and urine chemical tests revealed increased inflammatory markers and slightly increased cholestasis indices. Prednisone $37,5 \mathrm{mg} /$ day was started and tapered to $5 \mathrm{mg}$. This dosage was considered the minimum needed to control the disease. Another HRCT performed shows the complete clearing of the consolidated process with the persistence of some stellate bronchocentric consolidation especially in the apical lobes bilaterally. Last spirometry shows improvement of the obstructive impairment $\mathrm{FEV}_{1} 81 \%$ of the predicted, a reduction of the $\mathrm{FEV}_{1} / \mathrm{FVC}$ ratio at $56 \%$. The patient remains asymptomatic after 8 months of follow up.

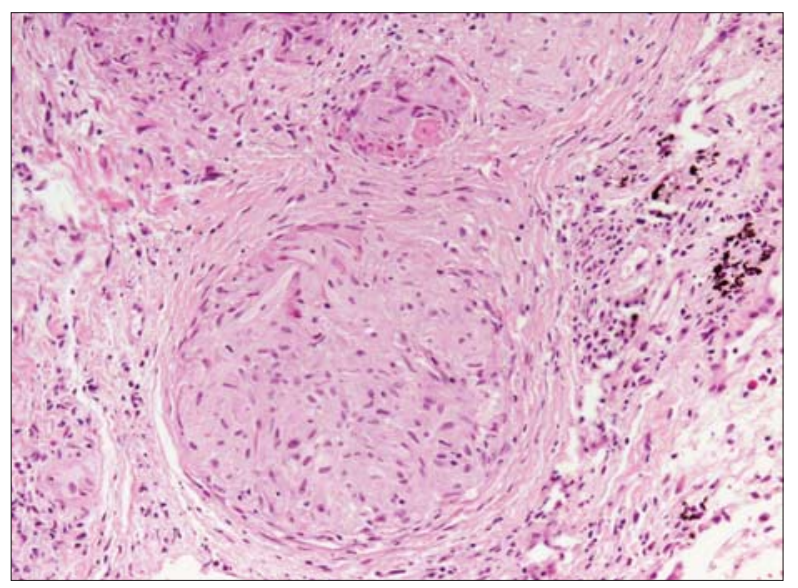

Fig. 3. - Higher magnification of the granulomas.

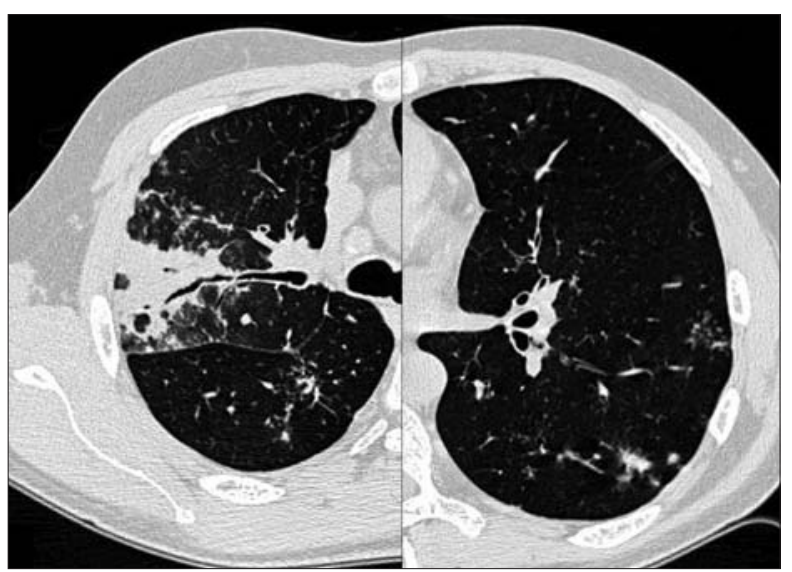

Fig. 5. - HRCT scan through the right hilar region (left) shows a new area of inhomogeneous consolidation in the right upper lobe, with air bronchogram inside, associated with peripheral small nodules, which appear to be mainly distributed along the bronchovascular bundles, under the costal pleura and the fissure. In the right lower lobe (right side) the HRCT demonstrates other small irregular nodules, distributed along the peripheral pulmonary vessels. 


\section{Discussion}

The histological findings together with the HRCT and the clinical follow up suggest in our case a relapsing OP in a patient with asthma and chronic sarcoidosis. A recent paper [1] confirms that in patients with interstitial lung disease, HRTC findings contribute with clinical history, physical examination findings and laboratory investigations to an overall impression, from which physicians subsequently modify, refine, or radically change a presumptive diagnosis and treatment plan. In our patient with HRCT findings of massive enlargement of the mediastinal lymph nodes and peripheral non calcified nodules distributed in the subpleural regions, the presence of an alveolar consolidation was suggestive of alveolar sarcoidosis but this last presentation is not specific and could be found in a large array of lung pathology. The differential diagnosis of this alveolar radiographic pattern of sarcoidosis includes fungal and mycobacterial infections, bronchoalveolar cell carcinoma, Wegener's and bronchocentric granulomatosis, focal radiation and drug-induced lung injury, lipoid pneumonia, alveolar proteinosis, and OP an inflammatory lung disease that primarily affects the small conducting airways and resulting from organisation of inflammatory exudates [2]. The three main characteristic imaging patterns of OP consist of multiple alveolar opacities (typical OP), solitary opacity (focal OP), and infiltrative opacities (infiltrative OP) [3]. Large thoracic adenopathy is not characteristic of OP. In our case, differential diagnosis comprised other clinical entities causing migratory alveolar opacity in asthmatic patients as chronic eosinophilic pneumonia (CEP), allergic bronchopulmonary aspergillosis (ABPA) and Churg Strauss vasculitis. Diagnostic steroid trials are best undertaken only when all clinical features conform to classic descriptions of CEP or, ideally, after confirming tissue eosinophilia. The histological confirmation of the suspected disease and the exclusion of differential diagnosis and especially of infectious triggers is otherwise always warranted. Transbronchial biopsy of the lung was performed in our patient and this technique is considered sufficient in most cases to make a differential diagnosis of both pulmonary sarcoidosis and OP $[4,5]$. The diagnosis of cryptogenic organising pneumonia (COP) requires the exclusion of several possible causes and/or specific context known to be occasionally associated with organising pneumonia that have been recently described in a review [6] such as connective tissue disease, various haematological disorders, malignancies, immunoglobulin deficiencies, inflammatory bowel disease and gastro-oesophageal reflux, thyroid diseases; it may also occur secondary to lung transplantation or bone marrow grafting, drug or be radiation induced. The clinical and imaging features of "secondary" organising pneumonia are similar to those of COP [7]. The most intriguing characteristic of OP, is its usual dramatic reversibility with corticosteroids as happens even in CEP and in ABPA.
Rodriguez et al. in 2001 reported an association between sarcoidosis and COP [8] not relapsing at steroid cessation with organising pneumonia at the periphery of granulomatous lesions. Minimal data exists concerning possible cause-effect relationship among three different diseases observed in our patient. Epidemiological studies recently demonstrated that sarcoidosis may be associated with a lower incidence of atopy and allergic disease [9]. Inverse association between sarcoidosis and atopic asthma has been described [10] and the cells, chemokine and cytokine involved in asthma are different respect to that playing a prominent pathogenic role in sarcoidosis $[11,12$, 13]. Obstructive pneumonia whatever its cause (e.g. foreign body inhalation) may comprise features of lipid pneumonia, chronic abscess and organising pneumonia, in our patient we observed migratory feature of the consolidated lesions but we cannot exclude the possibility that relapsing OP has been facilitated by bronchial obstructions due to sarcoidosis and chronic asthma. The role of the myofibroblast in wound healing and fibrocontractive diseases is critical [14]. Myofibroblast apoptotic activity is increased in the newly formed connective tissue in organising pneumonia [15]. Myofibroblast proliferation, fibrogenesis and matrix turnover is altered in the myofibroblasts demarcating granulomas and is significantly enhanced by LTD4 $[16,17]$. Therefore it is possible that in COP associated with persistent asthma and chronic sarcoidosis, myofibroblast apoptosis and activity is impaired leading to relapsing disease.

We confirmed the previously described role of mildly increased $\gamma \mathrm{GT}$ and alkaline phosphatase levels as predictor of COP relapse [18]. Some authors have proposed low doses of corticosteroids and a short duration of treatment for relapses, in order to avoid the side-effects of corticosteroids and treatment for long periods without necessity in patients who would not relapse [18]. Occasional spontaneous improvement of COP or response to treatment with antibiotics (especially macrolides) has been reported [19]. Radiological, functional and BAL derived markers of follow up might be useful to better understand this intriguing diseases and for the subsequent therapeutical decisions.

\section{References}

1. Aziz ZA, Wells AU, Bateman ED, et al. Interstitial lung disease: effects of thin-section CT on clinical decision making. Radiology 2006; 238: 725-33.

2. Lee FE, Caracta CF, Fine A, Berk JL. Fevers, weight loss, and bilateral peripheral infiltrates in a young man. Chest 1999; 115: 1181-1183.

3. Cordier JF, Loire R, Brune J. Idiopathic bronchiolitis obliterans organising pneumonia. Definition of characteristic clinical profiles in a series of 16 patients. Chest 1989; 96: 999-1004.

4. Azzam ZS, Bentur L, Rubin AH, Ben-Izhak O, Alroy G. Bronchiolitis obliterans organising pneumonia. Diagnosis by transbronchial biopsy. Chest 1993; 104: 1899-1901. 
5. Trisolini R, Lazzari Agli L, Cancellieri A, et al. Transbronchial needle aspiration improves the diagnostic yield of bronchoscopy in sarcoidosis. Sarcoidosis Vasc Diffuse Lung Dis 2004; 21: 147-51.

6. Cordier J-F. Cryptogenic organising pneumonia. Eur Respir J 2006; 28: 422-446.

7. Lohr RH, Boland BJ, Douglas WW, Dockrell DH, et al. Organising pneumonia. Features and prognosis of cryptogenic, secondary, and focal variants. Arch Intern Med 1997; 157: 1323-1329.

8. Rodríguez E, López D, Bugés J, Torres M. Sarcoidosisassociated bronchiolitis obliterans organising pneumonia. Arch Intern Med 2001; 161: 2148-2149.

9. Kokturk N, Han ER, Turktas H. Atopic status in patients with sarcoidosis. Allergy Asthma Proc 2005; 26: 121-4.

10. Grutters JC, Drent M, van Velzen-Blad H, van den Bosch JM. Inverse association between sarcoidosis and atopic asthma. Sarcoidosis Vasc Diffuse Lung Dis 1998; 15: 193-4.

11. Akbari O, Faul JL, Hoyte EG, et al. CD4+ Invariant TCell-Receptor+ Natural Killer T Cells in Bronchial Asthma. N Engl J Med 2006; 354: 1117-29.

12. Morgan AJ, Guillen C, Symon FA, et al. Expression of CXCR6 and its ligand CXCL16 in the lung in health and disease. Clin Exp All 2005; 35: 1572-82.
13. Agostini C, Cabrelle A, Calabrese F, et al. Role for CXCR6 and Its Ligand CXCL16 in the Pathogenesis of TCell Alveolitis in Sarcoidosis. Am J Resp Crit Care Med 2005; 172: 1290-1298.

14. Gabbiani G. The myofibroblast in wound healing and fibrocontractive diseases. J Pathol 2003; 200: 500-503.

15. Lappi-Blanco E, Soini Y, Paakko P. Apoptotic activity is increased in the newly formed fibromyxoid connective tissue in bronchiolitis obliterans organising pneumonia. Lung 1999; 177: 367-376

16. Fireman E, Schwartz Y, Mann A, Greif J. Effect of montelukast, a cysteinyl receptor antagonist, on myofibroblasts in interstitial lung disease. J Clin Immunol 2004; 24: 418-25.

17. Kaarteenaho-Wiik R, Sademies O, Paakko P, Risteli J, Soini Y. Extracellular matrix proteins and myofibroblasts in granulomas of sarcoidosis, atypical mycobacteriosis, and tuberculosis of the lung. Hum Pathol 2007 Jan; 38: 147-5.

18. Lazor R, Vandevenne A, Pelletier A, Leclerc P, CourtFortune I, Cordier JF. Cryptogenic organising pneumonia. Characteristics of relapses in a series of 48 patients. Am J Respir Crit Care Med 2000; 162: 571-577.

19. Epler GR, Colby TV, McLoud TC, Carrington CB, Gaensler EA. Bronchiolitis obliterans organising pneumonia. $N$ Engl J Med 1985; 312: 152-158.

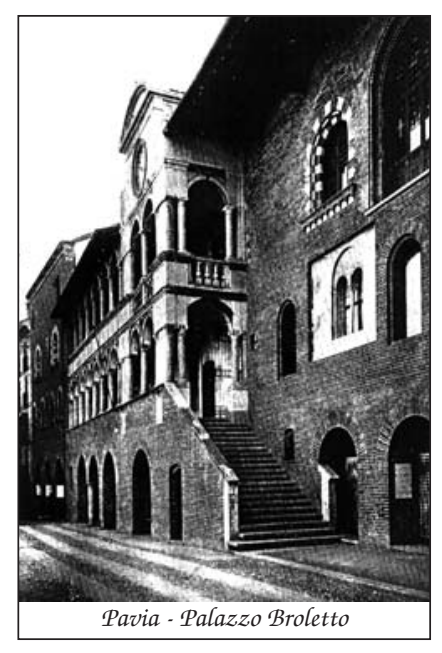

